\title{
ARGOS: a Fractioned Geosynchronous SAR
}

\author{
A Monti Guarnieri ${ }^{\mathrm{a}, *}$, O. Bombaci ${ }^{\mathrm{b}}$, T. F. Catalano ${ }^{\mathrm{b}}$, C. Germani ${ }^{\mathrm{b}}$, C. Koppel $^{\mathrm{c}}$, F. Rocca $^{\mathrm{a}}$, \\ G. Wadge ${ }^{\mathrm{d}}$ \\ ${ }^{a}$ Dipartimento di Elettronica, Informazione e Bioingegneria - Politecnico di Milano - IT \\ ${ }^{b}$ Thales Alenia Space Italia s.p.a. - Via Saccomuro, 24 - 00131 Roma - IT \\ ${ }^{c}$ Kopoos Consulting Ind - 57 Rue d'Amsterdam, 75008 Paris - FR \\ ${ }^{d}$ The University of Reading - Whiteknights, Reading RG6 2AB - UK
}

\begin{abstract}
A swarm of N Synthetic Aperture Radar (SAR) sensors in a geostationary orbit would provide all-day-all-weather imaging within a continental region, using direct downloading for real time data exploitation. The coherent combinations of the echoes would improve the signal-to-noise ratio by a factor of $\mathrm{N}^{2}$, leading to metric resolution, 20 - 40 minutes minimum observation time, multipolarimetric and interferometric imaging. Fast evolving events like landslides, floods, soil moisture changes, volcanic activity, co-seismic motions, infrastructure deformations and columnar water-vapor maps would be monitored continuously from space for the first time. The observed area would have a footprint up to a thousand kilometers wide, set using electronic steering, anytime, and anywhere within a continent. In this paper, we take advantage of the preliminary concept definition of the "Advanced Radar Geosynchronous Observation System" ARGOS, to provide applications, scalable extension from a single monostatic SAR to a constellation of active and passive sensors, discussion of launch strategies and Radar payload and comparison with concepts proposed in the literature.
\end{abstract}

(C) 2015 Published by Elsevier Ltd.

Keywords: swarm, fractioned observations, SAR, Interferometry, geostationary

\section{Introduction}

Geosynchronous Synthetic Aperture Radar (SAR) observation systems would be highly appreciated, as their capability of continuous observation would well suit their day and night functionality as well as their cloud penetration capability. Such unique monitoring

\footnotetext{
*Corresponding author ph: +390223993446; fax: $+390223993413$

Email address: monti@elet.polimi.it (A Monti Guarnieri ) URL:

http://www.deib.polimi.it/eng/people/details/282280

(A Monti Guarnieri )
}

capabilities have never been fully exploited, since the revisit time of typical Low Earth Orbit (LEO) SAR is in the order of several days, and increases up to weeks if the observation geometry has to be exactly replicated - that is the case for interferometry. The need for fast revisit, that is essential for applications for security, emergencies and intelligence led to the development of constellations, like the COSMO-SKYMED, SAR-Lupe, Sentinel-1, etc. Nonetheless, the continuous observation is still a goal far from present capabilities of such constellations.

To assess the size of such continuous observation constellation and the optimal orbit placement, let us first 
fix the maximum incidence angle $\theta_{i} \sim 40^{\circ}$, as shown in Fig. 1, on the left.
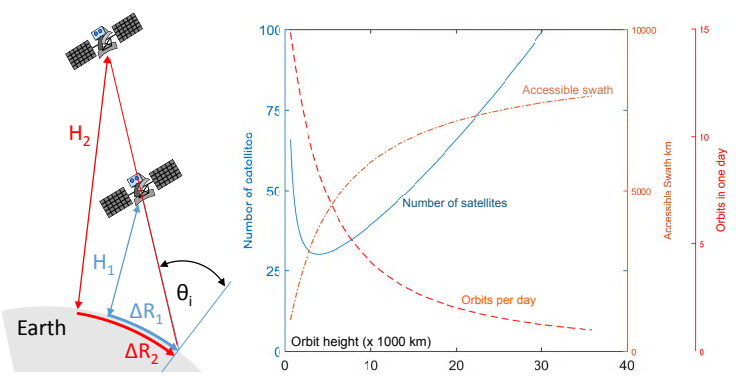

Figure 1. Left: geometry for computing swath width, $\Delta R$, at different sensor heights, by constraining the maximum incidence angle. Right: plots of the accessible swath width (dash-dot line), the number of orbits per day (dashed line), and the number of satellites needed for a one-hour revisit as function of the orbit height.

On one side, the higher the satellite, the larger would be the accessible swath, and then the shorter the revisit. However, we should account for the longer mean orbit time:

$$
T_{o}=2 \pi \sqrt{\frac{\left(H_{s}+R_{e}\right)^{3}}{\mu}}
$$

$R_{e}$ being the Earth radius and $\mu=3.986 \times 10^{14} \mathrm{~m}^{3} / \mathrm{s}^{2}$ the standard Earth gravitational parameter.

The dependence of the accessible swath and the number of orbits per day on the altitude, are plotted in Fig. 1 on the right, by assuming a swath of $800 \mathrm{~km}$ (that is $\pm 400 \mathrm{~km}$ and right/left looking capabilities) at a reference altitude of $700 \mathrm{~km}$. The number of satellites in an hypothetical constellation that provides global access with one hour revisit in the worst case (at the equator) is derived from (1):

$$
N_{s}=\frac{2 \pi R_{e} T_{o}}{\Delta R\left(H_{s}\right) \cdot 86400}
$$

That number of necessary satellites is $N_{s}=72$ at an orbital height of $1300 \mathrm{~km}$, i.e. the orbit proposed by Jet Propulsion Laboratory (JPL) in [1], and reduces to the minimum of $N_{s}=30$ at the orbit height of $4000 \mathrm{~km}$, low Medium Earth Orbit (MEO) orbit. Above that orbit, the reduction of velocity causes the number of satellite to increase.

However, we can show that a particular geosynchronous SAR swarm can achieve much shorter revisit time at the price of renouncing to world-wide coverage. Tomiyasu first proposed in [2, 3] a Geosynchronous Earth Orbit (GEO) SAR that acted like a plane flying daily from New York at noon to Buenos Aires at midnight and back, observing a strip about $4000 \mathrm{~km}$ wide with a spatial resolution of about $15 \mathrm{~m}$. The similar single satellite MEO payload proposed by JPL mentioned above, would require $45 \mathrm{~kW}$ radio frequency power and a $12 \mathrm{~m}$ diameter antenna to achieve enough SNR (Signal to Noise Ratio) in this short observation time [4]. These technologies are not available, as yet, and a cost of about 25 billions dollars was estimated at the time of proposal. Recently, several groups in the People's Republic of China have been working on this concept $[5,6,7]$. We call this concept $A$.

In parallel, a vastly cheaper (quasi) geostationary SAR, that could be piggybacked on telecommunication satellites has been studied since [8]. To ensure the link budget, while adopting a common $5 \mathrm{~m}$ diameter antennas and $1 \mathrm{~kW}$ transmitters, long coherent integration times and thus observation times up to 8 hours would be used. The variable atmospheric conditions during this time would impose corrections, to be able to focus the data $[9,10,11]$. However, to exploit the small motions of the quasi-geostationary platform transforms a drawback into an advantage. An on-board L-band Radar could measure the water vapor column over a continent providing a new image every 15 minutes at $1 \mathrm{~km}$ resolution, while an X-band Radar could operate in tandem on the very same platform and share the same antenna, watching any region within that continent.
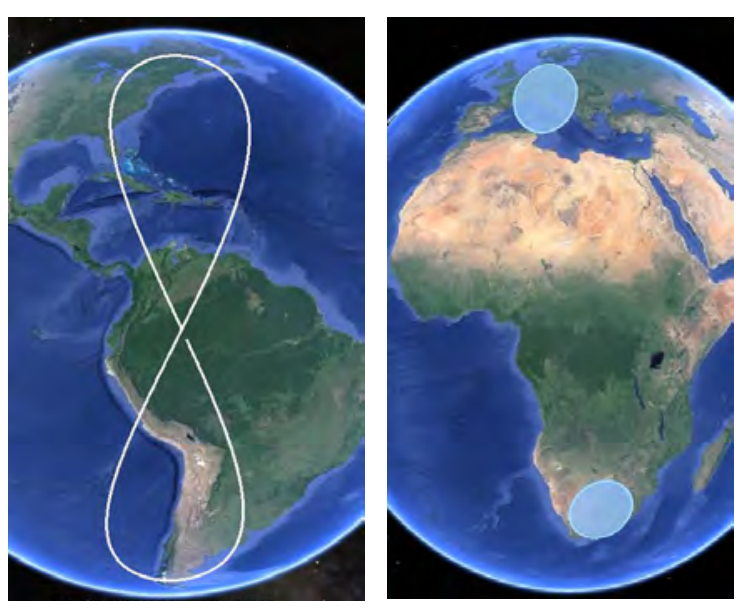

Figure 2. Left: nadir track for the $50^{\circ}$ inclination, 0.009 eccentricity geosynchronous SAR in [3], concept A. Right: two possible beams generated by an L-band quasi-geostationary SAR (eccentricity $<0.001$, no inclination) with a $7 \mathrm{~m}$ circular antenna, concept $\mathrm{B}$.

A study of this system, that we term concept $B$, is ongoing [12]. Indeed the costs have been confirmed to be much lower than $100 \mathrm{M} \$$, but the length of the observation time would only allow the recovery of the targets that are stable in this time interval to much less than a wavelength (centimeters), the so called Persistent 
Scatterers, that should be stable over periods of 8 hours, twice per day. However, billions of these exist, typically man-made objects, reinforced concrete, poles, exposed rocks, deserts, etc [13]. One could measure their motion to the millimeter twice a day, but the actual resolution of the other parts of the image would be limited due to their mechanical instability and thus the defocusing due to their motion. Indeed, the few minutes observation time needed for Concept A, coupled with the long Lband wavelength, would allow a much better imaging, but at a much greater cost. Concept $\mathrm{B}$ coverage is more limited than the continental scale of Concept A, but the possible targets access is the same, see Fig. 2.

A third concept has been recently introduced in [14], concept $C$, that is unique in achieving instantaneous, continental-scale access, and short revisit time, less than hourly, whilst keeping the resolution as fine as that of a LEO SAR. It comprises a fractioned swarm of N neighboring quasi-geostationary compact SAR's, all receiving not only their own signal (monostatic) but also the Radar signals (bistatic) coming from the other satellites. We shall see that such a N-inputs-N-outputs Multi Input Multi Output (MIMO) configuration is capable of generating $\left.N_{c}=N \times(N+1)\right) / 2$ virtual acquisitions $[15,16,17,18]$. This allows the progressive scan of a real $100 \mathrm{~km}$ wide antenna, to be completed in a fraction of the time needed by a single satellite, at $12 / N_{c}$ hours.

Therefore concept $\mathrm{C}$, with its continental access, shown in Fig. 2 on the right, competes with the worldwide coverage constellation of 30 LEO satellites in Fig. 1. We will show that such geostationary swarm gets the same revisit with just 6 satellites.

Further, to complete the comparison, we should account also for power and antenna size. We assume to fix the Signal-to-Noise-Ratio (SNR), the ground resolution $\rho_{a z} \times \rho_{r g}$ and the swath. We can express the Radar equation as follows:

$$
S N R=\frac{P_{m} A_{e}^{2} \eta_{T}}{4 \pi \lambda^{2} R^{4}} \sigma^{0} \rho_{\mathrm{az}} \rho_{\mathrm{rg}} \frac{T_{S}}{N_{0}}
$$

$P_{m}$ being the mean power, $\eta_{T}$ the total losses, $A_{e}$ being the antenna equivalent area, $\mathrm{R}$ the slant range, $\lambda$ the wavelength, $\sigma^{0}$ the backscatter coefficient, $N_{0}$ the noise spectrum density at the receiver and $T_{s}$ the synthetic aperture time, and $v$ is the sensor velocity:

$$
T_{s}=\frac{\lambda R}{2 v \rho_{a z}}
$$

This equation has been generalized into (A.1) in the appendix to hold the case of a swarm with $\mathrm{N}$ active and $\mathrm{M}$ passive sensors. The mean power, $P_{m}$, the antenna area, $A_{g}$, and the number of sensors to get one hour revisit (worst case), computed by combining (3) and (4) are compared for the LEO-MEO-GEO concepts in Tab. 1, by assuming to achieve a ground resolution of $10 \times 10 \mathrm{~m}$ (single look), a Noise Equivalent Sigma Zero (NESZ) of $-20 \mathrm{~dB}$ at beam center, a ground swath of $500 \mathrm{~km}$, and L-band. The noise figure has been assumed $3 \mathrm{~dB}$, and total losses $4 \mathrm{~dB}$ and antenna directivity (one-way) losses $1.2 \mathrm{~dB}$. The last three rows refer to concept $\mathrm{A}$ [1], concept $\mathrm{B}$ and concept $\mathrm{C}$ : in this very last case we assumed $\mathrm{N}=6, \mathrm{M}=2$. The resulting imaging and revisit time is actually less than one hour, and can be further improved by reducing resolution. The combination of this short revisit and the fractioning of power and antenna, [19], positions concept $C$ well ahead the 25 billions dollars monostatic geosynchronous concept A. Concept B is much cheaper, but has 7 hours image time and 24 hours revisit.

\begin{tabular}{|c|c|c|c|c|c|c|}
\hline Orbit & Altitude $\mathrm{km}$ & Access & Swath km & \# satellites & Mean power kW & Antenna $\mathrm{m}^{2}$ \\
\hline LEO & 630 & world & 500 , strip & 66 & 0.23 & \\
\hline low MEO & 4000 & world & 500 , strip & 30 & 0.55 & 42 \\
\hline GEO-synchronous (A) & 36000 & continental & 500, strip & 24 & 24 & 350 \\
\hline $\begin{array}{l}\text { GEO-stationary (B) } \\
\text { GEO-stationary (C) }\end{array}$ & $\begin{array}{l}36000 \\
36000\end{array}$ & $\begin{array}{l}\text { continental } \\
\text { continental }\end{array}$ & $\begin{array}{l}2100 \times 1200 \\
2100 \times 1200\end{array}$ & $6+2$ & $\begin{array}{l}2.2 \\
0.5^{*}\end{array}$ & $\begin{array}{l}33 \\
33^{*}\end{array}$ \\
\hline
\end{tabular}

Table 1. LEO-MEO-GEO SAR swarms comparison (L-band)

The applications addressed by such continuous monitoring system are discussed in section 2. Section 3 introduces the main mission components. The swarm configurations are detailed in section 4 and 5, whereas the payload, platform, launcher accommodation and orbit placement and maintenance are discussed in section 6 and 7. In the appendix, we discuss the choice of the wavelength to maximize the signal-to-noise ratio.

\section{Prequels to the swarm}

The geosynchronous swarm, ARGOS, first introduced in [14], is made possible using three concepts that were already discussed in the open literature, that we wish here to acknowledge. The capability of acting both as a transmitter and a receiver from many other satellites corresponds to the Multiple Channel Architecture MIMO concept introduced for LEO-SAR [15, 26, 27]. There it was shown how a partitioned antenna could be implemented. The availability of many phase centers was then exploited to densify the along track sampling and then getting larger swaths, or to increase range resolution [28, 29]. ARGOS takes advantage of this densification along its elliptical, periodic orbit to reduce the image and revisit time.

The Interferometric Cartwheel [30, 31] is a swarm of three passive satellites and one active illuminator, a Single Input Multiple Ouptut (SIMO) system. The orbit 
ellipticity is similar to that of ARGOS, though not in an Earth fixed frame. This makes the observed scene continuously changing, the revisit is in the order of days, as usual for LEO-SAR.

The third concept, the "P-Band Distributed SAR" in [19], is a combination of the previous two: it is a MIMO, getting the full $\mathrm{N}^{2}$ gain in number of phase centers $[17,18]$, but implemented as a constellation in LEO orbit - like the cartwheel. The objective was to fraction the huge antenna required by a P-band SAR, while keeping the swath large. The reuse of the very same transmission frequency was already there, although the way different sources could be separated in the angular domain, [14], was not discussed. A further full MIMO swarm was introduced by Ender et. al. in $[15,16]$ dealing with detection of ships and their velocity estimation.

The idea is to exploit the geostationary orbit to combine the advantages of the three concepts, resulting in a unique system capable of providing short revisit imaging (thanks to MIMO and the orbit), continental access (thanks to the orbit) and fine resolution (thanks to the swarm). There is no other system capable of achieving these together.

\section{Applications}

In order to be able to appreciate the advantages of such a system, we introduce a list of applications, the type of users addressed, and the type of measurements achievable. The list is given in Tab. 3: mention is made to evidence the uniqueness that the system would bring in any specific field, as for [12]. The multimode, multibeam, multifrequency, multipolarization features of a modern SAR sensor are still possible by this concept, allowing the search for the best compromise for users needs. In addition to that, the users applications will take advantage from the swarm composition of active/passive sensors both in the "staring" as well as in the "sliding" configurations to be discussed in the following sections.

\section{Mission structure}

The possible components of a fractioned geosynchronous SAR swarm are represented in Fig. 3. There, an example of a constellation with $\mathrm{N}=2$ active sensors and $M=1$ passive one is shown. The observations allow to generate two monostatic images, as the number of active sensors, and further three bistatic images, i.e. all the pairs of transmitter and receiver non colocated. The bistatic images are practically identical to those that
Table 2. Geosynchronous swarm: applications and uniqueness

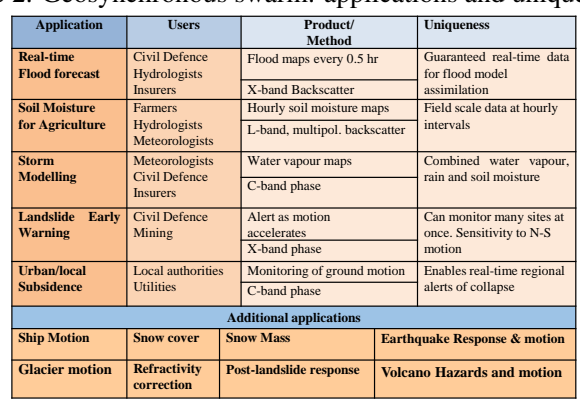

would be produced by a monostatic system positioned half way between the transmitter and the receiver: the location of the correspondent phase centers are marked as crosses in the figure $[15,16,17,18]$.

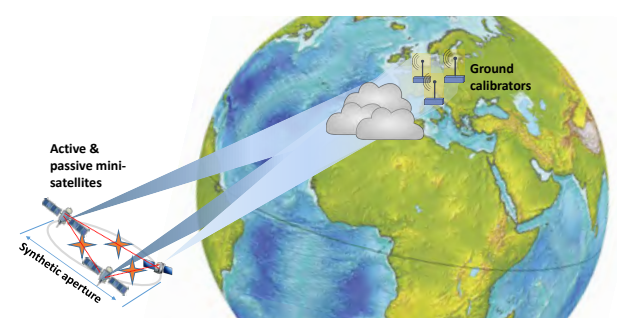

Figure 3. Components of the proposed geosynchronous SAR swarm:. Two active sensors and one passive are represented. The crosses represent the additional virtual equivalent monostatic phase centers.

The synthesis of the SAR antenna (azimuthal resolution) is carried out by imaging the target from different angles, that correspond now to the positions of the phase centers along the orbit. Any additional phase center with its motion shortens the time needed to sample the synthetic antenna (typically $100 \mathrm{~km}$ wide) at all positions. Thus, the total time needed to sample everywhere the antenna with at least one phase center is vastly reduced.

A network of ground calibrators is also represented in the figure, to be used to compensate for the atmospheric and ionospheric phase screen, clock jitter and precise orbit $[20,21,22]$. As a geostationary constellation is always in view of the scene, this leads to two further benefits:

- the calibration can be performed by placing just a few compact active transponders

- data are downloaded directly to the ground with no time-lag, leading to potential real-time applications. 
However, the achievement of such system is conditional on some enabling technologies, in particular:

- the electric propulsion, that allows the mass and space reduction needed to fit multiple satellites within one launch,

- compact, lightweight Radar payload, capable of delivering peak power in the order of few $\mathrm{kW}$ and antenna size less than ten meters, both one order of magnitude less than in concept $\mathrm{A}$,

- launchers capable of placing the whole swarm of six mini-satellites in Geosynchronous Transfer Orbit (GTO), or an incremental build-up, maintaining and expanding the constellation by LEO and then transfer into GEO.

\section{Staring and sliding configurations}

In this section we discuss how to optimize the positions of the satellites in order to have optimal distributions of the phase centers in space as well as in time. Thus, we achieve the scan of the $100 \mathrm{~km}$ wide antenna by exploiting the apparent motion of all the phase centers, minimizing the coverage losses. In [14], two different constellations topologies were identified by the set of parameters, $\left\{t_{n}, \psi_{n}, e_{n}\right\}$ describing the time of the perigee, the central longitude and the eccentricity for each of the satellite's elliptical orbit in Earth Centered Earth Fixed (ECEF) reference. Those orbits can be approximated by their polar coordinates in the equatorial plane:

$$
\begin{aligned}
r(t) & =R_{\text {geo }}\left(1-e_{n} \cos \left(\Omega_{E}\left(t-t_{n}\right)\right)\right) \\
\phi(t) & =\psi_{n}+2 e_{n} \sin \left(\Omega_{E}\left(t-t_{n}\right)\right)
\end{aligned}
$$

$\Omega_{E}$ being the Earth angular velocity.

There are two different configurations of interest, that we define here as "staring" and "sliding", that improve the "concentric" and "iso-elliptical" configurations introduced in [14].

In the staring configuration the goal is to achieve an uniform spectral coverage with time: the entire full width of the antenna is progressively scanned at all times. The result from numerical optimizations shows that the relevant parameters are perigee times $t_{n}$, whereby the eccentricity and the longitude can be kept fixed. The optimization assumed as a goal the minimization of the spectral holes in a fixed time interval, all around the orbit. In the six active and two passive systems shown in Fig. 4, the total of $N \times(N+1) / 2+M \times$ $N=33$ phase centers results in an average of $80 \%$ of the antenna sampling being covered in 30 minutes (see bottom-right panel), while a longer interval is needed to cover the whole angular spectrum. The top panel in the figure shows the tracks spanned within 30 minutes by the real and the virtual phase centers in the equatorial plane, where the longitude is along the horizontal. We remind that the orbits of the real phase centers are the vectors $\vec{S}_{n}$ expressed by (5), whereas each virtual phase center is placed midway between two real phase centers: $\vec{S}_{n, m}=\left(\vec{S}_{n}+\vec{S}_{m}\right) / 2$. The total aperture spanned, represented in grey shades, is in that case more than $70 \%$ of the total aperture (the extremes of the ellipsis).

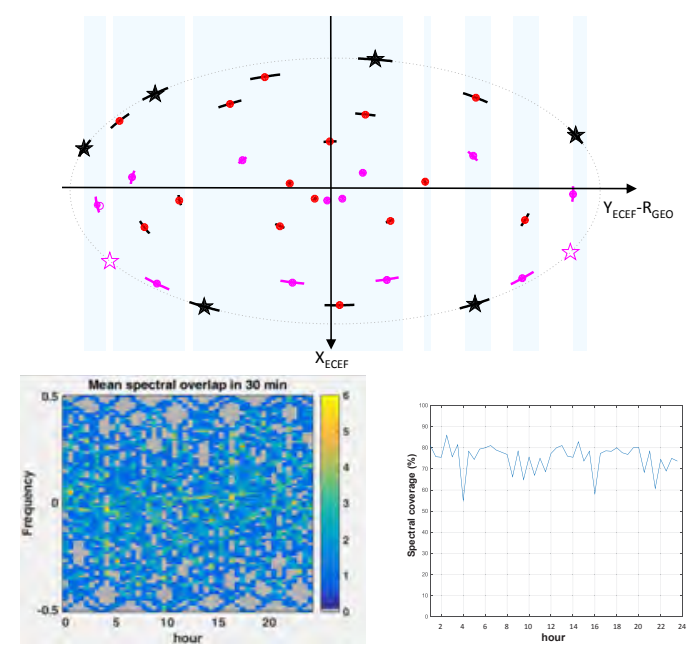

Figure 4. Optimal staring configuration. Upper graph: tracks of the of $\mathrm{N}=6$ active satellites (black stars) and $\mathrm{M}=2$ passive (pink stars) and of their virtual phase centers in 30 minutes, in the equatorial, ECEF reference (horizontal axis is longitude). Lower graph: angular spectral coverage with the time of the day (one orbit). Left: number of hits for each angle (Doppler frequency) in the assigned time interval. Right: percentage of coverage at any time during the 24 hour period.

The second concept is the sliding configuration. The idea is to achieve a shorter image time by keeping the formation tight, instead than uniformly spreading it as for the "staring" swarm. In that case, the total aperture is limited in the short term, and the resolution worsens, but a fine resolution can be achieved upon completion of an entire orbit, that occurs after one day. The "sliding" configuration is a generalization of the "isoelliptical" concept in [14]. In this case, the full set of orbit parameters $\left\{t_{n}, \psi_{n}, e_{n}\right\}$ in (5) for all the sensors (but one), has been optimized, aiming to the maximization of the spectral coverage within two orbital intervals of six hours displaced by twelve hours. The resulting spectral coverage, achieved by the six active sensors swarm in 30 minutes shown in Fig. 5 compares with the one achieved by the six active and two passive sensors in the 
staring configuration shown in Fig. 4. However, in this case the short term image has an azimuthal resolution coarser than what can be achieved in 24 hours.
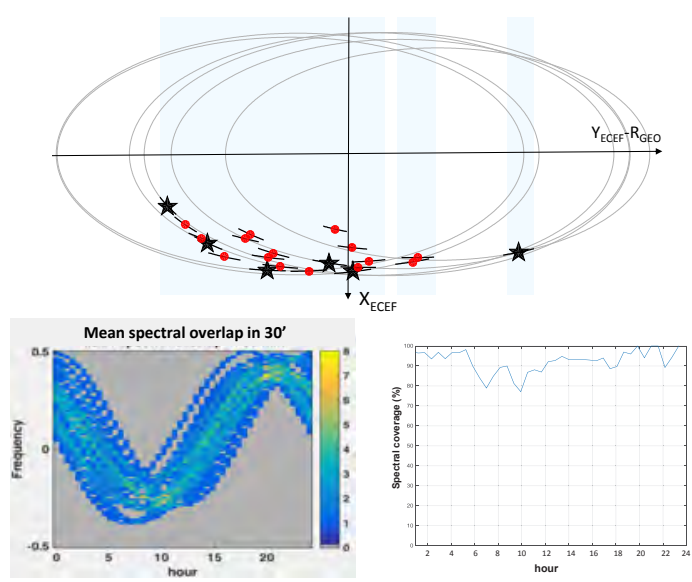

Figure 5. Optimal sliding configuration. Upper graph: tracks of the of $\mathrm{N}=6$ active satellites (black stars) and of the corresponding virtual phase centers in 30 minutes, in the equatorial, ECEF reference (horizontal axis is longitude). Lower graph: spectral coverage with the time of the day (one orbit). Left: for each frequency, right: overall percentage.

The choice between staring, sliding, and maybe other concepts is then a matter of applications. The staring swarm gets a uniform aperture span and antenna coverage along the orbit, in the 24 hours, yet with some voids. In the staring swarm here assumed, the coverage ranges from $65 \%-80 \%$, quite uniformly distributed in the 24 hours time of a single orbit. In contrast, the sliding swarm spectral coverage is more compact, giving a much shorter imaging time: in the example, $30 \mathrm{~min}-$ utes, with less than $20 \%$ spectral voids. However, the instantaneous antenna width spanned is half of the total, getting coarse resolution in short term and fine at the end of the day. This configuration is better for capturing fast evolving surfaces and events, such as flooding, landslides or volcanic eruptions. As a final remark we notice that the precision required for orbit keeping is loose, as for image reconstruction. The compensation of deviation from ideal orbit can in fact be handled in processing, like for airborne [23] and LEO-space borne system [24], whereas the baseline decorrelation in interferometry is avoided by keeping the orbit tube well below the critical value [25]:

$$
B_{n c}=\left|\frac{\lambda R \tan \left(\theta_{i}\right)}{2 \rho_{r g}}\right|,
$$

Such value ranges from hundred of meters to several kilometers for the configuration of interest.

\section{Launcher, platform and Radar payload}

The implementation of ARGOS as a swarm leaves freedom degrees to be exploited, depending on the costbenefit trade-offs and the progression of investments. One could start with a single mini-satellite, that is actually option B, and then add further active and/or passive systems. Alternatively, a swarm of six satellites can be stowed, and then simultaneously launched, in a single Ariane-5.

In order to define potential mission scenarios we propose here three configurations of interest, represented in Fig. 6:
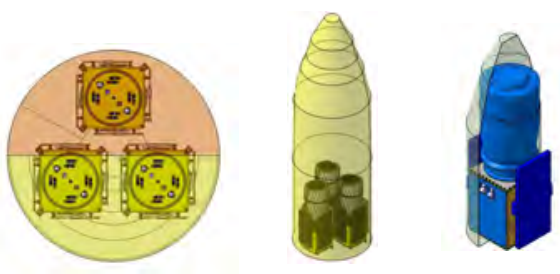

Figure 6. Launchers configurations in the Ariane and VEGA cases

- A multiple launch with a single Ariane 5, with 6 active space-crafts staggered $3+3$ in the launch vehicle fairing.

- A multiple launch with a single Ariane 5, with 3 active space-crafts and $\mathrm{N}$ mini-passive, the 3 active being placed on top launch vehicle deck and the $\mathrm{N}$ mini-passive in the bottom deck. In case of passive instrument, different configuration of PRIMA (Piattaforma Riconfigurabile Italiana Multi-Applicazione) [34, 35] can be considered, for a class up to $500 \mathrm{~kg}$ of satellite to be launched with Ariane 5 in multiple launches or in a single launch with VEGA (Vettore Europeo di Generazione Avanzata).

- A single launch of 1 space-craft at time with VEGA.

Both active and passive solutions take advantage of an Electric Propulsion system (EP) capable to guarantee the autonomous orbit raising from classical GTO (Ariane 5) or LEO VEGA to GEO, that is discussed in a later section. Orbit control needs in terms of formation flying performance and orbit maintenance are vital. The usage of a GNSS receiver will permit highly autonomous orbit control capability and autonomous operational routine phase and maintenance. 


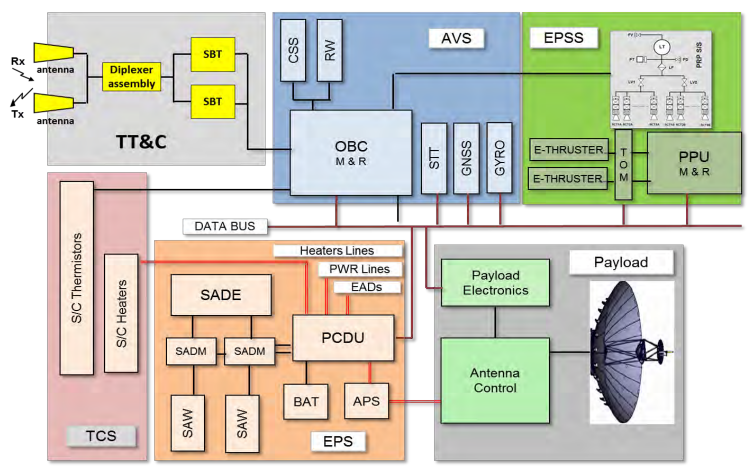

Figure 7. Block scheme of the Radar payload integrated in the platform

The block scheme of the Radar payload, the major component in the system, is shown in Fig. 7 as integrated in the platform (the PRIMA is used in this example). The Radar should provide beam scanning capabilities within a continental access region. Fig. 8 shows examples of X-band footprints obtained by a 7 meter antenna, to be compared with the L-band shown in Fig. 2. The significant reduction in the footprint allows for a much finer resolution, if needed, whereas the wide coverage can still be achieved (at the price of power or resolution) by scanning multi-beams in a ScanSAR fashion $[32,33]$. In the $\mathrm{X}$-band case shown in the figure, the beam-width scales by a factor 8 , projecting a ground footprint of about $150 \times 260 \mathrm{~km}$. This gives a power gain factor of 64 with respect to the wide coverage shown in Fig. 2, that can be exploited to reduce the resolution cell to $3 \times 3$ meters and at the same time to simultaneously scan 6 beams.

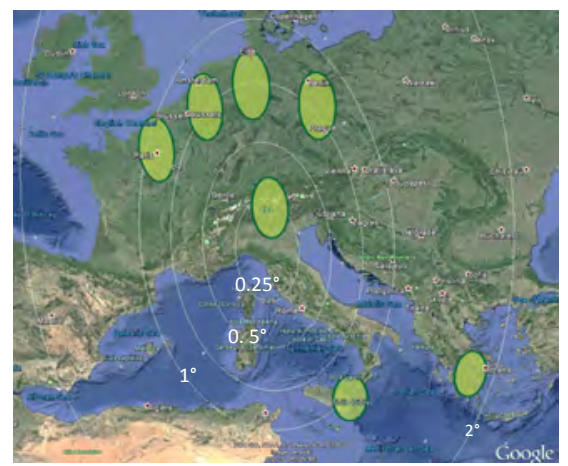

Figure 8. Example of high resolution X-band footprints.

The Radar payload to achieve this flexible pointing, and, at the same time to be compact and lightweight, should use a foldable or unfurlable large reflector, a feed array sub-system, and a programmable digital chirp generator to achieve a flexible bandwidth, like the one in $[34,35]$. The key technology to enable continental access, and at the same time wide bandwidth for fine resolution, is a reduced active feed array, whose performances is magnified by the stable reflector architecture, but with a modifiable feed system operating frequency and related design. The large number of transmitting modules in the feed array would ensure at once high reliability figure and graceful degradation of the performance.

The functional diagram of the SAR architecture sketches a high level perspective of its functionalities and of their allocation within the two main subsystems of the sensor, like for LEO-SAR: the SAR Electronics Subsystem (SES) and SAR Antenna Subsystem (SAS): the scheme is shown in Fig. 9. The design is suitable for all the frequencies: L, S, C, and X. The architecture is also usable for the design of the passive payload in which only the receiver section can be studied to comply with dedicated interferometric applications' requirements.

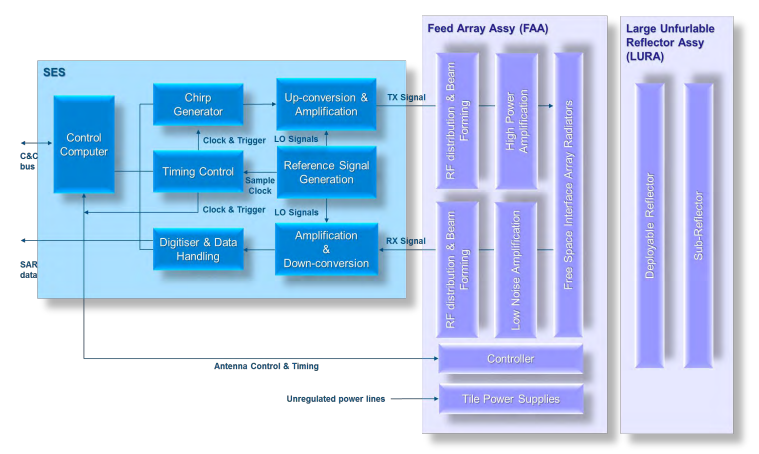

Figure 9. SAR functional diagram

\section{Orbit placing and electric propulsion}

The advantage of Electric Propulsion (EP) is that it consumes one fifth to one tenth of the propellant used by classical chemical propulsion. Without EP, we wouldn't be able to launch the whole six-satellite constellation at once. Moreover, the thrust is proportional to the power, so because in general the power on board is limited, only low thrust propulsion can be practically achieved. In contrast, for Radar satellites, the power to mass ratio is much higher than for example, for commercial communication satellites. This favors the use of EP for the proposed concepts, particularly for performing the orbit transfer between GTO or LEO to the final 
GEO previously discussed, because during the transfer the Radar payload is switched off, leaving the electric propulsion with the highest level of power inducing a higher thrust than for other COMSAT. This is attractive for shortening the transfer (particularly from LEO orbit), whereby the mass and volume saving coming from electric propulsion enables multiple payloads in a single launch.

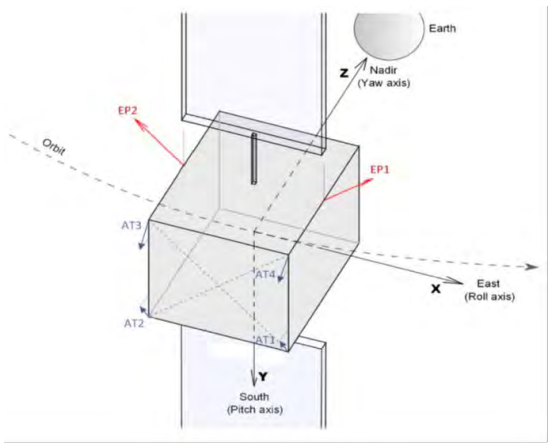

Figure 10. Satellite configuration with the thrusters for both propulsion and attitude control.

The satellite configuration with the thrusters is sketched in Fig. 10 with some adaptations from [40]. First the two main thrusters sets are EP1 and EP2: those are designed for performing the North-South and EastWest station keeping around the ascending node of the orbit: they provide thrust component along the South so that they are suited for the correction of inclination around that ascending node. They also have components of thrust toward East and West, so they are able to correct the East-West acceleration shift: a simple adjustment of the ratio of use EP1 with respect to the total North-South correction (EP1+EP2) duration will enable East-West correction during each North-South correction (the delta V East-West represents only one tenth of the delta V North-South). Moreover the configuration is also able to perform any need of station re-positioning toward East, by using EP2 then after some cost using EP1 for canceling the drift velocity or toward the West by using EP1 then EP2 (in order to become more efficient, without losing the North-South correction induced, those maneuvers should only occur around the ascending node $\pm 90^{\circ}$ ). In addition, one of those main thrusters set EP1 or EP2 (or both alternatively) is used for performing the orbit transfer: in order to have the thrust aligned with the optimal thrust direction for the orbit transfer, the pitch axis (Y) has to be inclined. A rotation of the satellite around the thrust axis is then performed in order to get the solar array axis perpendicular to the Sun vector and to maximize electrical power from the Sun. Such attitude was followed for example by the European probe Smart-1, [41], for the transfer from Earth to Moon. The thrusters sets AT1 to AT4 are dedicated for the 3 axis attitude control of the satellite: together AT1 and AT2 provide torque around the roll axis, and together AT2 and AT3 provide torque around the pitch axis. The yaw axis is controlled by the use of AT1 and AT3. The use of those thrusters induces a small force along the yaw axis for every attitude control: because the yaw axis is also the Earth radius, it is easy to correct by firing up to 4 thrusters at a location on the orbit opposed to the previous point (12 hours later), but because attitude controls are occurring in every points of the orbit, that force will be averaged to a null force along every orbit, so that no secular trend will be observed.

\subsection{Orbit transfers}

Two cases of orbit transfers are considered: first a classic case from Super-GTO to GEO (already experimented in flight with two satellites launched on March 1, 2015 by SpaceX's Falcon 9 rocket with ABS 3A and EUTELSAT 115 West B all-electric satellites to a supersynchronous transfer orbit), and a second case more demanding at satellite level, but less demanding at launcher level is a starting point with a circular orbit in LEO.

Super-GTO to GEO. The 3D plot of the orbit transfer is given in Fig. 11 on the left, along with the projections from each axis. The initial orbit is inclined at $7^{\circ}$ with perigee of $200 \mathrm{~km}$ and apogee of $61000 \mathrm{~km}$. The initial mass is $1000 \mathrm{~kg}$, the thrusters specific impulse is $1600 \mathrm{~s}$ (according to the use of Hall effect thrusters). The electrical power used is $6 \mathrm{~kW}$ for a maximum thrust of $0.33 \mathrm{~N}$. With such a relatively high thrust the transfer is performed in 69 days, including eclipse periods where no thrust is provided. The delta $\mathrm{V}$ of the orbit transfer maneuver is $2095 \mathrm{~m} / \mathrm{s}$, that is a propellant mass consumption of $125 \mathrm{~kg}$.

LEO to GEO. The 3D plot of the orbit transfer is given in Fig. 11 on the right. The starting orbit is circular $700 \mathrm{~km}$ inclined at $7^{\circ}$. The initial mass is 1000 $\mathrm{kg}$, the thrusters specific impulse is $1600 \mathrm{~s}$ (Hall effect thrusters) [42]. The electrical power used is $6 \mathrm{~kW}$ for a maximum thrust is $0.33 \mathrm{~N}$. With such thrust the spiral transfer is performed in 166 days (including the eclipses periods where no thrust is provided). The delta $\mathrm{V}$ of the orbit transfer maneuver is $4627 \mathrm{~m} / \mathrm{s}$, that is a propellant mass consumption of $256 \mathrm{~kg}$. 


\subsection{North-South East-West station keeping}

With a thruster cant angle of $45^{\circ}$ with respect to the North-South axis, the delta $\mathrm{V}$ needed for maintaining the inclination at $0.1^{\circ}$ and for 10 years of operations is the order of $707 \mathrm{~m} / \mathrm{s}$ (the East-West station keeping being performed for free taking into account the thrust components along the East-West axis) thus the mass consumption is respectively of $36 \mathrm{~kg}$.

The attitude control is needed to keep the antenna pointed to the scene, particularly when small coveragehigh resolution is needed. The active attitude control is driven by a cost constant of:

$$
K_{D B}=\frac{T_{i y}}{I} \frac{\alpha_{1}}{\AA^{2}}
$$

where $T_{i y}$ is the total torque impulse needed for 1 year (in $\mathrm{Nms} / \mathrm{year}$ ) for each axis, $I$ the inertia (in $\mathrm{kg} \times \mathrm{m}^{2}$ ), $\alpha_{1}$ the control angle accuracy and $\stackrel{\circ}{\alpha}$ the angular velocity measurable limit of the dead band cycle. From [43], $K_{D B}$ is about $700 \mathrm{kN} \times \mathrm{s}^{3} /(\mathrm{kg} \times \mathrm{m} \times$ year $\times \mathrm{deg})$ which gives, for $\alpha_{1}=0.1^{\circ}$ and $\stackrel{\circ}{\alpha}=0.001^{\circ} / \mathrm{s}$, a total torque impulse of $100 \mathrm{kN} \times \mathrm{m} \times \mathrm{s}$ for 3 axis control for 10 years and hence finally a mass consumption of $3 \%$ of the initial mass (with Hall effect thruster technology). The perturbing torques due to sun pressure as well as the thruster misalignments are not included in the above cost, but those can be minimized by proper design. Electric propulsion will be implemented for performing the tasks of propulsion and attitude control, while removing the need of reaction wheels, because the attitude pointing is less constraining to $0.1^{\circ}$.

From a GEO nominal orbit, the de-orbiting is performed by small impulses placing the out-of-use spacecraft at $300 \mathrm{~km}$ above GEO, at a cost much lower than that of $1 \mathrm{~kg}$ of propellant $(0.03 \%$ of the initial mass $)$.

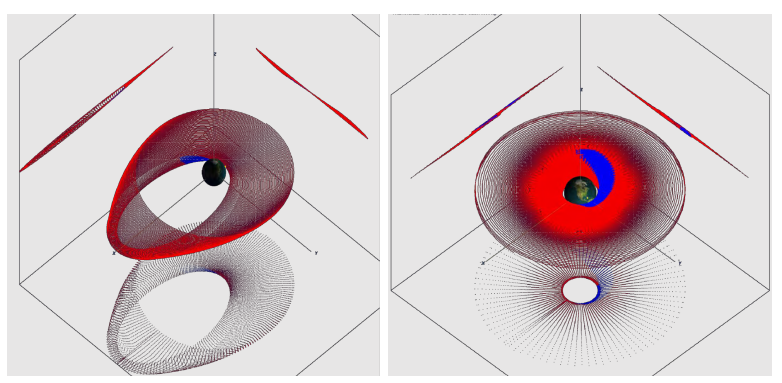

Figure 11. Orbit transfer: left, Super-GTO to GEO and, right. LEO to GEO.

\section{Conclusions}

A geosynchronous SAR concept has been discussed. This concept gathers the advantages provided by (i) the geostationary orbit, (ii) the SAR, (iii) the swarm, detailed in Fig. 12. The system inherits the all-time-allweather benefits of a SAR, but is remarkably endowed with a resolution that scales inversely with the squared number of satellites. The geosynchronous location naturally solves the down-link bottleneck enabling a raft of applications and services for emergencies and security. Such a system has been termed ARGOS, after the allseeing, never-sleeping, giant guardian of Greek myth.

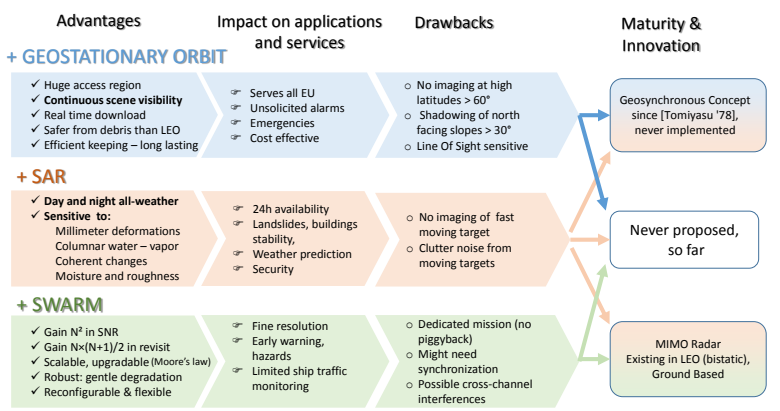

Figure 12. Benefits and drawbacks of the proposed approach.

\section{Appendix A. On the optimal wavelength}

The choice between frequency, say from L-band to Xband, should satisfy the different user needs [12]. However, the huge path losses and the consequent long integration times required for geostationary SAR, causes thermal and clutter noises to be the major limitation for many applications.

The aim of this appendix is to compare performances in terms of signal-to-noise ratio for different wavelengths. We assume that the antenna area is fixed to a maximum (that depends on the available technology, cost, size and weight), and that also the ground swath covered is fixed to the value in Tab. 1. This implies that, for the shorter wavelengths, more swaths need to be scanned cyclically and this compensates exactly for the increased antenna directivity, making NESZ independent on the wavelength. However, SNR depends upon the backscatter, $\sigma^{0}$, that changes with wavelength [39]. The SNR can be expressed by applying to (3) the gain coming for the $\mathrm{N}$ active satellites and the $\mathrm{M}$ passive [14]:

$$
S N R=\frac{P_{m} A_{e} \eta_{T}^{2}}{4 \pi \lambda_{L}^{2} R^{4}} \sigma^{0}(\lambda) \rho_{\mathrm{az}} \rho_{\mathrm{rg}} \frac{T_{S}}{N_{0}}\left(N^{2}+N M\right)
$$

This expression does not account for target decorrelation in the long integration time, that is clutter noise. 
To this end, we can exploit the empirical Intrinsic Clutter Model (ICM) by Billingsley [36, 37]. The power spectrum due windblown Radar clutter as a function of clutter Doppler, $f_{D}=-2 v / \lambda$, is:

$$
P\left(f_{D}\right)=\frac{\alpha}{\alpha+1} \delta\left(f_{D}\right)+\frac{1}{\alpha+1} \frac{\lambda \beta}{4} \exp \left(\frac{\lambda \beta}{2}\left|f_{D}\right|\right)
$$

the first addendum being the power of the stable component (the signal), and the second the contribution of the moving one (the clutter noise). The two parameters involved, $\alpha$ and $\beta$ are expressed by the empirical relations:

$$
\begin{aligned}
& \alpha=489.9 \cdot w^{-1.55} \cdot f_{0}^{-1.21} \\
& \beta=\frac{1}{0.1048\left(\log _{10} w+0.4147\right)}
\end{aligned}
$$

where $w$ is the wind speed in miles per hour, $f_{0}$ the carrier frequency in $\mathrm{GHz}$, and $\beta$ is in meters per second. Furthermore, the clutter noise should be combined with thermal noise, leading to a signal-to-total-noise ratio $S N_{T} R$ :

$$
S N_{T} R=\left(\left(S N R \cdot \frac{\alpha}{\alpha+1}\right)^{-1}+S C R^{-1}\right)
$$

where Signal-to-Clutter-Noise can be evaluated, for geosynchronous SAR as from [38]:

$$
S C R=\frac{\alpha}{1-\exp \left(-\frac{v_{s} \beta \lambda}{2 L_{e}}\right)}
$$

$L_{e}$ being the equivalent antenna length. The total SNR is plotted in Fig. A.13 by assuming Ulaby and Dobson "Grasses" and "Shrubs" classes [39], the latter corresponding to the case that has been adopted for computing performances shown in Tab. 1. Notice that the absence of any wind favors the shorter wavelength, particular for the case of vegetation, due to the reflection from leaves that are almost invisible in L-band, but rather bright in X-band. However, for moderate and strong breeze, the decorrelation is responsible of significant losses in $\mathrm{X}$ - and $\mathrm{Ku}$-band, that is no more preferable in those cases. This loss is expected to be much stronger, in the real case, since the ICM model in $[36,37]$ is too optimistic for very long integration times [38].

The results support a preference for C-band for wideswath, coarse resolution observations. On the other hand, X-band would be preferred for spot beams over coherent targets, like man made objects - mainly in urban areas - and exploiting household satellite TV antenna as reflectors of opportunity. In the case of ARGOS, however, either C- or X-band should be considered.
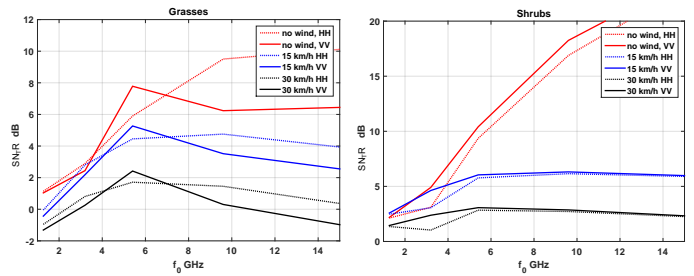

Figure A.13. Total singal to thermal and clutter noise, $S N_{T} R$, evaluated for "grasses" (left) and "shrubs" (rigth) classes [39], and different wind speed as function of the frequency.

[1] S. Madsen, C. Chen, W. Edelstein, Radar options for global earthquake monitoring, in: Geoscience and Remote Sensing Symposium, 2002, IGARSS '02, IEEE International, pp. 14831485

[2] K. Tomiyasu, Tutorial review of synthetic-aperture Radar (SAR) with applications to imaging of the ocean surface, Proceedings of the IEEE 66 (5) (1978) 563-583.

[3] K. Tomiyasu, J. L. Pacelli, Synthetic aperture Radar imaging from an inclined geosynchronous orbit, IEEE Transactions on Geoscience and Remote Sensing 21 (3) (1983) 324 -329.

[4] W. N. Edelstein, Concepts and technologies for synthetic aperture Radar from MEO and geosynchronous orbits, Proceedings of SPIE 5659 (2005) 195-203.

[5] C. Hu, Z. Liu, T. Long, An improved CS algorithm based on the curved trajectory in geosynchronous SAR, IEEE Journal of Selected Topics in Applied Earth Observations and Remote Sensing 5 (3) (2012) 795-808.

[6] G.-c. Sun, M. Xing, Y. Wang, A 2-D Space-Variant Chirp Scaling Algorithm Based on the RCM Equalization and Subband Synthesis to Process Geosynchronous SAR Data, 52 (8) (2014) 4868-4880.

[7] M. Jiang, W. Hu, C. Ding, G. Liu, The Effects of Orbital Perturbation on Geosynchronous Synthetic Aperture Radar Imaging, 12 (5) (2015) 1106-1110.

[8] C. Prati, F. Rocca, D. Giancola, A. Monti Guarnieri, Passive geosynchronous SAR system reusing backscattered digital audio broadcasting signals, IEEE Transactions on Geoscience and Remote Sensing 36 (6) (1998) 1973-1976.

[9] D. Bruno, S. Hobbs, Radar imaging from geosynchronous orbit: Temporal decorrelation aspects, IEEE Transactions on Geoscience and Remote Sensing 48 (7) (2010) 2924 -2929.

[10] L. Iannini, A. Monti Guarnieri, Atmospheric phase screen in ground-based Radar: Statistics and compensation, Geoscience and Remote Sensing Letters, IEEE PP (99) (2010) 536 -540.

[11] S. Hobbs, C. Mitchell, B. Forte, R. Holley, B. Snapir, P. Whittaker, System design for geosynchronous synthetic aperture Radar missions, IEEE Transactions on Geoscience and Remote Sensing 52 (12) (2014) 750-7763.

[12] G. Wadge, A. Guarnieri, S. Hobbs, D. Schultz, Potential atmospheric and terrestrial applications of a geosynchronous Radar, in: Geoscience and Remote Sensing Symposium (IGARSS), 2014 IEEE International, 2014, 946-949.

[13] D. Perissin, A. Ferretti, Urban-target recognition by means of repeated spaceborne SAR images, IEEE Transactions on Geoscience and Remote Sensing 45 (12) (2007) 4043-4058.

[14] A. Monti Guarnieri, A. Broquet as, A. Recchia, F. Rocca, J. Ruiz-Rodon, Advanced Radar geosynchronous observation system: Argos, Geoscience and Remote Sensing Letters, IEEE 12 (7) (2015) 1406-1410.

[15] J. Ender, A Concept for a Spaceborne Multistatic Radar Sys- 
tem for SAR/GMTI and Jammer Reconnaissance, NATO Sensors and Electronics Technology (SET) research panel Symposium, Warsaw, May 2001.

[16] D. Cerutti-Maori, J.H.G. Ender, Performance analysis of multistatic configurations for spaceborne GMTI based on the auxiliary beam approach, in: Radar, Sonar and Navigation IEE Proceedings, 13 April 2006, Vol.153, no.2, 96-103

[17] D. Tarchi, F. Oliveri, P. F. Sammartino, MIMO Radar and ground-based SAR imaging systems: Equivalent approaches for remote sensing, IEEE Transactions on Geoscience and Remote Sensing 51 (1) (2013) 425-435.

[18] G. Krieger, MIMO-SAR : Opportunities and Pitfalls 52 (5) (2014) 2628-2645.

[19] G. Fasano, M. D’Errico, G. Alberti, S. Cesare, G. Sechi, Distributed Space Missions for Earth System Monitoring, Springer, 2013, Ch. P-Band Distributed SAR.

[20] J. Gonzalez, M. Bachmann, G. Krieger, H. Fiedler, Development of the tandem-x calibration concept: Analysis of systematic errors, IEEE Transactions on Geoscience and Remote Sensing 48 (2) (2010) 716-726.

[21] G. Krieger, M. Younis, Impact of Oscillator Noise in Bistatic and Multistatic SAR, IEEE Geoscience and Remote Sensing Letters 3 (3) (2006) 424-428.

[22] M. Rodriguez-Cassola, P. Prats, P. Lopez-Dekker, G. Krieger, A. Moreira, General Processing Approach for Bistatic SAR Systems : Descrip- tion and Performance Analysis, in: EUSAR. 10th European Conference on, 2010, pp. 998-1001.

[23] S. N. Madsen, H. A. Zebker and J. Martin, Topographic mapping using Radar interferometry: Processing techniques, IEEE Transactions on Geoscience and Remote Sensing, 31(1), Jan 1993, 246-255.

[24] D. D'Aria, A. M. Guarnieri, High-Resolution Spaceborne SAR Focusing by SVD-Stolt, Geoscience and Remote Sensing Letters, IEEE, Oct 2007, vol.4, no.4, 639-643

[25] J. Ruiz-Rodon, A. Broquetas, E. Makhoul, A. Monti Guarnieri, F. Rocca, Nearly Zero Inclination Geosynchronous SAR Mission Analysis With Long Integration Time for Earth Observation, IEEE Transactions on Geoscience and Remote Sensing, Oct 2014, vol 52 (10), 6379-6391

[26] A. Goodman, S. Lin, D. Rajakrishna, J. Stiles, Processing of multiple-receiver spaceborne arrays for wide area SAR, IEEE Transactions on Geoscience and Remote Sensing 40 (4) (2002) 841-852.

[27] P. Lombardo, F. Colone, D. Pastina, Monitoring and surveillance potentialities obtained by splitting the antenna of the COSMOSkyMed SAR into multiple sub-apertures, IEE Proc.- Radar Sonar Navig 153 (2) (2006) 104-116.

[28] D. Cristallini, D. Pastina, P. Lombardo, Exploiting MIMO SAR potentialities with efficient cross-track constellation configurations for improved range resolution, IEEE Transactions on Geoscience and Remote Sensing 49 (1) (2011) 38-52.

[29] D. Pastina, F. Santi, M. Bucciarelli, MIMO distributed imaging of rotating targets for improved 2-D resolution, Geoscience and Remote Sensing, IEEE Letters, jan 2015, 190-194.

[30] D. Massonnet, Capabilities and limitations of the interferometric cartwheel, in: CEOS SAR Workshop, ESA-CNES, Toulouse, France, 26-29 October 1999

[31] D. Massonnet, E. Thouvenot, S. Ramongassie, L. Phalippou, A wheel of passive Radar microsats for upgrading existing SAR projects, in: International Geoscience and Remote Sensing Symposium, Honolulu, Hawaii, 24-28 July 2000.

[32] A. P. Luscombe, Taking a broader view RadarSAT adds ScanSAR to its operations, in: International Geoscience and Remote Sensing Symposium, Vol. 2, 1988, pp. 1027-1032.

[33] A. Currie, M. A. Brown, Wide-swath SAR, IEE Proceedings F
139 (2) (1992) 123-135.

[34] C. Germani, M. Veredice, A. Torre, Compact SAR: A novel Xband instrument, in: EUSAR 2014; 10th European Conference on Synthetic Aperture Radar; Proceedings of, 2014

[35] M. L'Abbate et.al., Compact SAR and micro satellite solutions for earth observation, in: 31st Space Symposium, Technical Track, Colorado Springs, Colorado USA, Apr 2015

[36] J. B. Billingsley, J. F. Larrabee, Measured spectral extent of 1and $\mathrm{x}$ - Radar reflections from windblown trees, Tech. rep., MIT Lincoln Laboratory, DTIC AD-A179942, (feb 1987).

[37] J. B. Billingsley, Exponential decay in windblown Radar ground clutter Doppler spectra: multifrequency measurements and model, Tech. rep., MIT Lincoln Laboratory, (jul 1996).

[38] A. Recchia, A. M. Guarnieri, A. Broquetas, A. Leanza, Impact of scene decorrelation on Geosynchronous SAR data focusing, IEEE Transactions on Geoscience and Remote Sensing, 2015, pp. 1-12, doi: 10.1109/TGRS.2015.2486385

[39] F. T. Ulaby, M. C. Dobson, Handbook of Radar Scattering Statistics for Terrain, Artech House, Norwood, 1989.

[40] M. Leomanni, A. Garulli, A. Giannitrapani, F. Scortecci, Precise attitude control of all-electric GEO spacecraft using xenon microthrusters, in: Proc. $33^{r d}$ International Electric Propulsion Conference IEPC, 2013.

[41] D. E. A. Milligan, Operationally enhanced electric propulsion performance on electrically propelled spacecraft, in: Proc. $29^{\text {th }}$ International Electric Propulsion Conference IEPC, 2015.

[42] C. R. Koppel, Method and a system for putting a space vehicle into orbit, using thrusters of high specific impulse, United States Patents US6116543 and US6213432.

[43] C. R. Koppel, Cubesat attitude and orbit control with 4 pulsed plasma thrusters, in: Atelier - Journee d'etude sur le controle d'attitude d'un nanosatellite, 2014. 